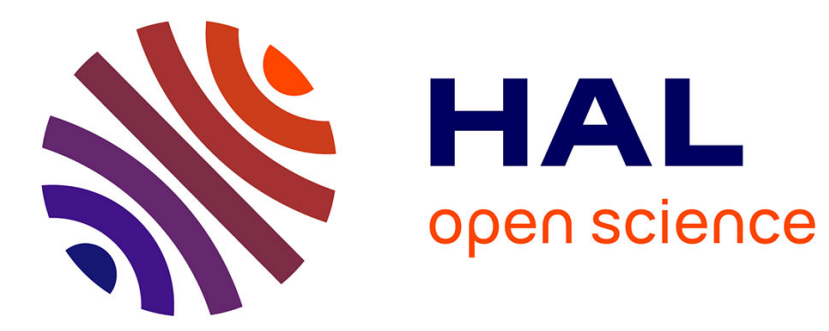

\title{
Engineering of mathematical chaotic circuits
}

René Lozi

\section{To cite this version:}

René Lozi. Engineering of mathematical chaotic circuits. Advances in Intelligent Systems and Computing, 2013, 210, pp.17-29. hal-00809583v2

\section{HAL Id: hal-00809583 \\ https://hal.science/hal-00809583v2}

Submitted on 9 May 2013

HAL is a multi-disciplinary open access archive for the deposit and dissemination of scientific research documents, whether they are published or not. The documents may come from teaching and research institutions in France or abroad, or from public or private research centers.
L'archive ouverte pluridisciplinaire HAL, est destinée au dépôt et à la diffusion de documents scientifiques de niveau recherche, publiés ou non, émanant des établissements d'enseignement et de recherche français ou étrangers, des laboratoires publics ou privés. 
Nostradamus 2013: Prediction, Modeling and Analysis of Complex Systems, Advances in Intelligent Systems and Computing, I. Zelinka, G. Chen, O. E. Rössler, V. Snasel, A. Abraham (Eds.) ,Springer, Vol. 210, 2013, pp. 17-29.

\title{
Engineering of mathematical chaotic circuits
}

\section{René Lozi}

Laboratoire J.A. Dieudonné, UMR CNRS 7351, Université de Nice-Sophia Antipolis,

Parc Valrose, 06108 NICE Cedex 02, France.

rlozi@unice.fr, tel. 33453755008.

\begin{abstract}
We introduce the paradigm of chaotic mathematical circuitry which shows some similarity to the paradigm of electronic circuitry especially in the frame of chaotic attractors for application purpose (cryptography, generic algorithms in optimization, control, ...).
\end{abstract}

Keywords: Chaos dynamics inside soft computing algorithms; Mathematical chaotic circuits

\section{Introduction}

The purpose of this communication is to build an analogous of paradigm of electronic circuitry, which is the design of electronic circuit: the paradigm of chaotic mathematical circuitry, in order to improve easily the performance of well known chaotic attractors for application purpose (cryptography, generic algorithms in optimization, control, ... ).

An electronic circuit is composed of individual electronic components, such as resistors, transistors, capacitors, inductors and diodes, connected by conductive wires through which electric current can flow. The combination of components and wires allows various simple and complex operations to be performed: signals can be amplified, computations can be accomplished, and data can be moved from one place to another. We introduce in the same way mathematical circuits which are composed of individual components (generators, couplers, samplers, mixers, and reducers, ...) connected through streams of data. The combination of such mathematical components leads to several news applications such as improving the performance of well known chaotic attractors (Hénon, Chua, Lorenz, Rössler, ...) for application purpose (chaotic cryptography, evolutionary and genetic algorithms in optimization, control,...). In Sec. 2 we present the symbols we introduce 
in the paradigm of mathematical circuits (generators, couplers, samplers, mixers, and reducers). In Sec. 3 we consider the design of two circuits, the first one for Chaotic multistream PseudoRandom Number Generators, the second one for Noise-resisting cryptographic transmitter and receiver. The conclusion is given in Sec. 4.

\section{Elementary components of mathematical circuits}

Analog circuits are very commonly represented in schematic diagrams, in which wires are shown as lines, and each component has a unique symbol. We present in this section the first symbols we design in order to draw mathematical schematic diagrams.

\subsection{Generator}

The first class of symbol we describe, generator symbols, are, from a mathematical point of view, equivalent to a battery or a current generator in electronic circuit. However we consider that they generate a numerical signal (in one or several dimensions) rather than a voltage or an intensity variation (nonetheless, a voltage or intensity variation can be considered as a physical signal which can be discretized). This signal can be either continuous, as in Chua's circuit, Lorenz or Rössler attractors or discrete as in Hénon or logistic map.

\subsubsection{Chua's circuit: a prototype for continuous generator}

Nowadays there is a worldwide tradition of use of the circuit invented by L.O. Chua in October 1983 for several purposes [2]. This circuit of Fig. 1(a) contains three linear energy-storage elements (an inductor and two capacitors), a linear resistor, and a single nonlinear resistor, namely, Chua's diode (Fig. 1(b)) with three segment linear characteristic defined by

$$
f\left(v_{R}\right)=m_{0} v_{R}+\frac{1}{2}\left(m_{l}-m_{0}\right)\left[\left|v_{R}+B_{p}\right|-\left|v_{R}-B_{p}\right|\right]
$$

where the slopes in the inner and the outer regions are $m_{0}$ and $m_{0}$, respectively, and $\pm B_{p}$ denotes the breakpoints. 
The dynamics of Chua's circuit is governed by Eq. (2) where $V_{C_{1}}, V_{C_{2}}$, and $I_{L}$ are respectively the voltages across the capacitors $C_{1}$ and $C_{2}$, and the intensity of the electrical current through the inductor $L$.

$$
\left\{\begin{array}{l}
C_{1} \frac{d v_{C_{1}}}{d t}=G\left(v_{C_{2}}-v_{C_{l}}\right)-f\left(v_{C_{1}}\right) \\
C_{2} \frac{d v_{C_{2}}}{d t}=G\left(v_{C_{1}}-v_{C_{2}}\right)+i_{L} \\
L \frac{d i_{L}}{d t}=-v_{C_{2}}
\end{array}\right.
$$

Equation (2) can be transformed into the third-order autonomous differential equation whose dimension-less form is

$$
\left\{\begin{array}{l}
\dot{x}=\alpha(y-x-f(x)), \\
\dot{y}=x-y+z \\
\dot{z}=-\beta y
\end{array}, \quad f(x)=b x+\frac{1}{2}(a-b)[|x+1|-|x-1|]\right.
$$

The parameter value

$$
\alpha=15.60, b=28.58, a=-\frac{1}{7} \text { and } b=\frac{2}{7}
$$

are very often used in order to generate chaotic signal. Even if the scheme of Fig. 1(a) is easily understandable for an electronics engineer, it is of no help to build a device using mathematical properties of chaos (like a secure communication system based on it [6]). This is why it is more useful to represent Chua's circuit like a chaos generator by the diagram of Fig. 2(a).

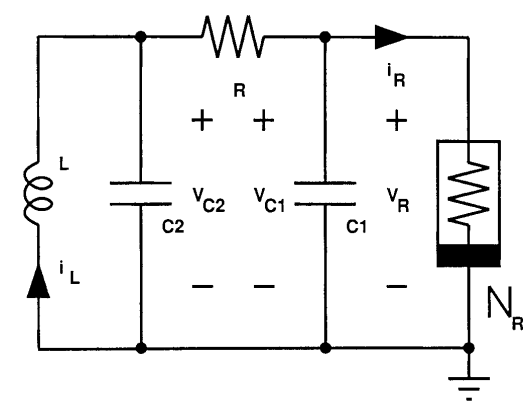

(a)

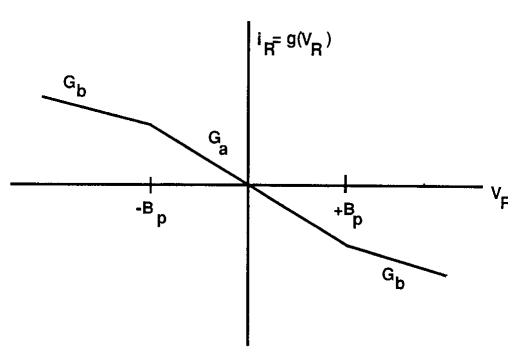

(b)

Fig. 1(a). Realization of Chua's circuit. (b). Three-segment piecewise-linear $v-i$ characteristic of nonlinear voltage controlled resistor (Chua's diode). 
On this expanded symbol of continuous generator, the solid line arrows coming out from the generator represent the three components of the signal $\underline{x}(t)=(x(t), y(t), z(t))$, the dashed line arrow points at $\lambda$ which stands for the parameter value defined by Eq. (4) and the dot line arrow points at $\underline{x}_{0}=\underline{x}(0)$ the given initial value of the signal.

If there is no ambiguity on the nature of the generator used, the symbol can be simplified as in Fig. 2(b).

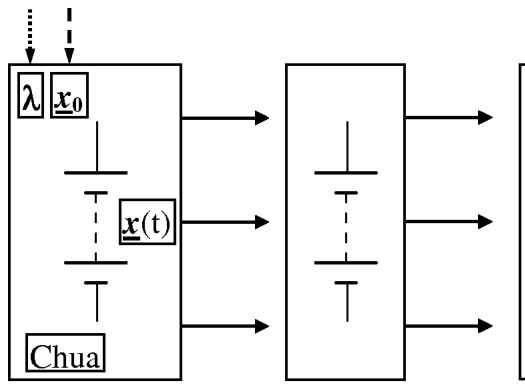

(a)

(b)

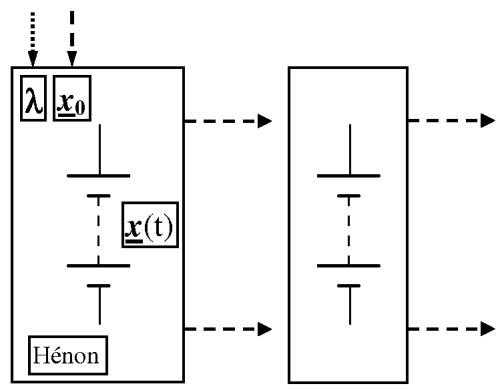

(d)

Fig. 2(a). Chua's circuit: continuous generator. (b). Simplified symbol of continuous generator, (c). Discrete generator (Hénon map, expanded symbol). (d). Simplified symbol discrete generator.

\subsubsection{Discrete generator: Hénon and Logistic map}

We need also to design chaotic circuitry for discrete signal. For this purpose some classical generators can be considered: in dimension 2, the Hénon map [4] defined by

$$
H_{a, b}:\left(\begin{array}{l}
x \\
y
\end{array}\right)=\left(\begin{array}{l}
y+1-a x^{2} \\
b x
\end{array}\right)
$$

with

$$
a=1.4, \quad b=0.3
$$

which must be associated to the dynamical system

$$
\left\{\begin{array}{l}
x_{n+1}=y+1-a x_{n}^{2} \\
y_{n+1}=b x_{n}
\end{array}\right.
$$


in order to provide a stream of chaotic numbers (Figs. 2(c) and 2(d)). And in 1dimension, the logistic map

$$
f(x)=4 x(1-x)
$$

associated to the dynamical system

$$
x_{n+1}=r x_{n}\left(1-x_{n}\right)
$$

Thereafter, another 1-dimensional chaotic generator, the symmetric tent map, will be, also, represented by the same symbol of Fig. 3 .

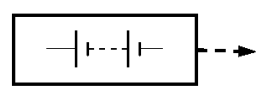

Fig. 3. 1-dimensional generator (logistic or tent map).

Remark: In the rest of this article, we use solid line arrow for continuous signal $x(t)$, and dashed line arrow for discrete signal $x_{n}$.

\subsection{Coupler}

The experimental observation of hyperchaotic attractors in open and closed chain of Chua's circuit was reported in 1994 [5]. The layout of the five identical coupled Chua's circuit forming a ring is displayed on Fig. 4.

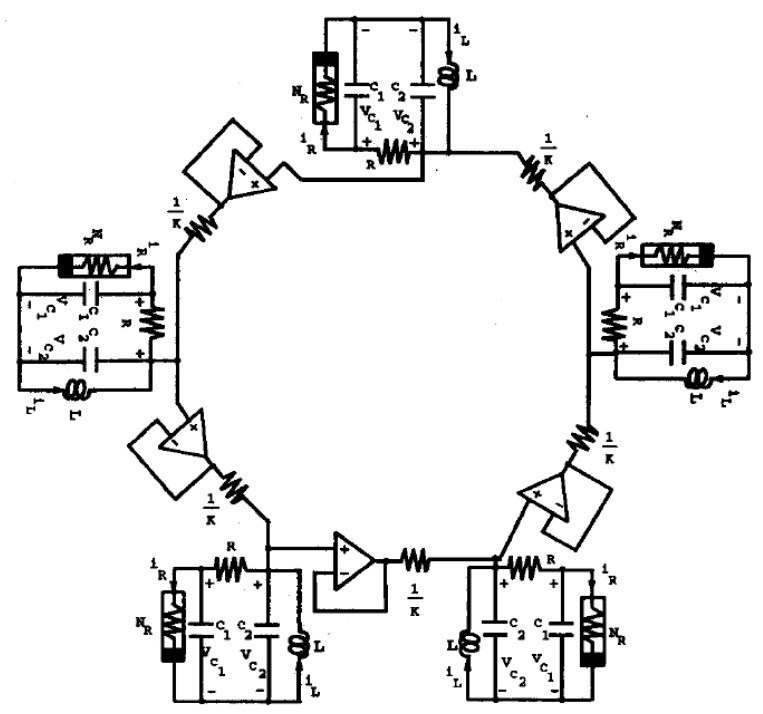


Fig. 4. Five identical coupled Chua's circuits forming a ring.

The state equations of this circuit are as follows (10). Identifying the symbols $\left(V_{C_{1}}^{(i)}, V_{C_{2}}^{(i)}, I_{L}^{(i)}\right)$ in each Chua's circuit with $\left(x^{i}, y^{i}, z^{i}\right)$, the state equations of the circuit can be translated into the differential equations (11), and the electronic circuit is symbolized by the mathematical circuit of Fig. 5 .

$$
\left\{\begin{array}{l}
C_{1} \frac{d v_{C_{1}}^{(1)}}{d t}=G\left(v_{C_{2}}^{(1)}-v_{C_{1}}^{(1)}\right)-f\left(v_{C_{1}}^{(1)}\right), \\
C_{2} \frac{d v_{C_{2}}^{(1)}}{d t}=G\left(v_{C_{1}}^{(1)}-v_{C_{2}}^{(1)}\right)+i_{L}^{(1)}+K_{1}\left(v_{C_{2}}^{(2)}-v_{C_{2}}^{(1)}\right) \\
L \frac{d i_{L}^{(1)}}{d t}=-v_{C_{2}}^{(1)}, \\
C_{1} \frac{d v_{C_{1}}^{(2)}}{d t}=G\left(v_{C_{2}}^{(2)}-v_{C_{1}}^{(2)}\right)-f\left(v_{C_{1}}^{(2)}\right), \\
C_{2} \frac{d v_{C_{2}}^{(2)}}{d t}=G\left(v_{C_{1}}^{(2)}-v_{C_{2}}^{(2)}\right)+i_{L}^{(2)}+K_{2}\left(v_{C_{2}}^{(3)}-v_{C_{2}}^{(2)}\right) \\
L \frac{d i_{L}^{(2)}}{d t}=-v_{C_{2}}^{(2)}, \\
C_{1} \frac{d v_{C_{1}}^{(5)}}{d t}=G\left(v_{C_{2}}^{(5)}-v_{C_{1}}^{(5)}\right)-f\left(v_{C_{1}}^{(5)}\right) \\
C_{2} \frac{d v_{C_{2}}^{(5)}}{d t}=G\left(v_{C_{1}}^{(5)}-v_{C_{2}}^{(5)}\right)+i_{L}^{(5)}+K_{5}\left(v_{C_{2}}^{(1)}-v_{C_{2}}^{(5)}\right) \\
L \frac{d i_{L}^{(5)}}{d t}=-v_{C_{2}}^{(5)},
\end{array}\right.
$$

In this figure the double rounded arrows symbolize the coupling of one Chua's circuit to the next one. In order to represent the coupling between mathematical equation, depending on the nature of the coupling, we can use two different symbols: the ring coupler corresponding to the coupling of one generator to the next one (Fig. 5) and the full coupler when the coupling involves more connections between the couplers (right hand side of Fig. 6). 


$$
\left\{\begin{array}{l}
\dot{x}^{1}=\alpha\left(y^{1}-x^{1}-f\left(x^{1}\right)\right), \\
\dot{y}^{1}=x^{1}-y^{1}+z^{1}+k_{1}\left(y^{2}-y^{1}\right), \\
\dot{z}^{1}=-\beta y^{1}, \\
\dot{x}^{2}=\alpha\left(y^{2}-x^{2}-f\left(x^{2}\right)\right), \\
\dot{y}^{2}=x^{2}-y^{2}+z^{2}+k_{2}\left(y^{3}-y^{2}\right), \\
\dot{z}^{2}=-\beta y^{2}, \\
\vdots \\
\dot{x}^{5}=\alpha\left(y^{5}-x^{5}-f\left(x^{5}\right)\right), \\
\dot{y}^{5}=x^{5}-y^{5}+z^{5}+k_{5}\left(y^{1}-y^{5}\right), \\
\dot{z}^{5}=-\beta y^{5},
\end{array}\right.
$$

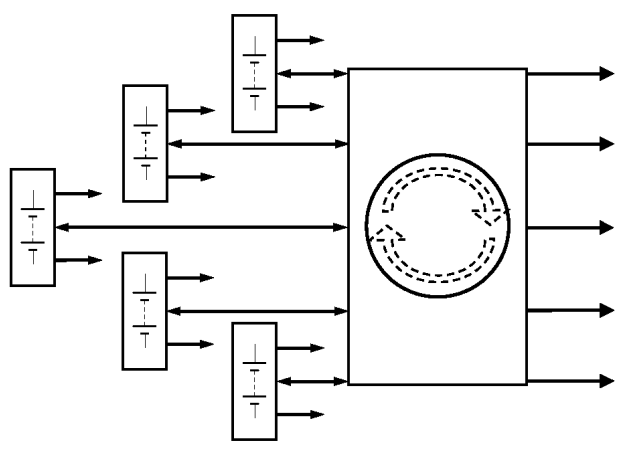

Fig. 5. Numerical circuit corresponding to the electronic circuit of Fig. 4.

It has been shown, few years ago [7] that the ultra-weak coupling of several logistic or symmetric tent maps

$$
f(x)=1-2|x|, \quad x_{n+1}=f\left(x_{n}\right)
$$

allows the production of long series of chaotic numbers equally distributed over the interval $[-1,1]$ of the real line.

The system of $p$-coupled tent map is given by

$$
X_{n+l}=F\left(X_{n}\right)=A \cdot\left(\underline{f}\left(X_{n}\right)\right)
$$

where

$$
X_{n}=\left(\begin{array}{c}
x_{n}^{1} \\
\vdots \\
x_{n}^{p}
\end{array}\right), \underline{f}\left(X_{n}\right)=\left(\begin{array}{c}
f\left(x_{n}^{1}\right) \\
\vdots \\
f\left(x_{n}^{p}\right)
\end{array}\right) \text { and }
$$




$$
A=\left(\begin{array}{ccccc}
\varepsilon_{1,1}=1-\sum_{j=2}^{j=p} \varepsilon_{1, j} & \varepsilon_{1,2} & \cdots & \varepsilon_{1, p-1} & \varepsilon_{1, p} \\
\varepsilon_{2,1} & \varepsilon_{2,2}=1-\sum_{j=1, j \neq 2}^{j=p} \varepsilon_{2, j} & \cdots & \varepsilon_{2, p-1} & \varepsilon_{2, p} \\
\vdots & \ddots & & \vdots & \vdots \\
\vdots & & \ddots & \vdots & \vdots \\
\varepsilon_{p, 1} & \cdots & \cdots & \varepsilon_{p, p-1} & \varepsilon_{p, p}=1-\sum_{j=1}^{j=p-1} \varepsilon_{p, j}
\end{array}\right)
$$

The design of the corresponding mathematical circuit is displayed on Fig. 6.

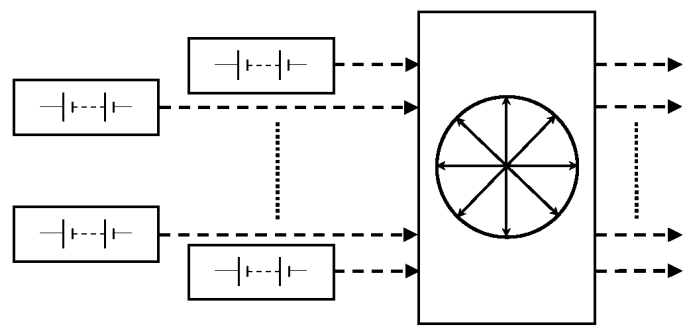

Fig. 6. Circuit of ultra-weak coupling of $p$ 1-dimensional chaotic maps.

\subsection{Sampler}

However chaotic numbers are not pseudo-random numbers because the plot of the couples of any component $\left(x_{n}^{l}, x_{n+1}^{l}\right)$ of iterated points $\left(X_{n}, X_{n+1}\right)$ in the corresponding phase plane reveals the map $f$ used as one-dimensional dynamical systems to generate them via Eq. (13). Nevertheless we have recently introduced a family of enhanced Chaotic Pseudo Random Number Generators (CPRNG) in order to compute very fast long series of pseudorandom numbers with desktop computer [9]. This family is based on the ultra-weak coupling mechanism which is improved in order to conceal the chaotic genuine function.

In order to hide $f$ of Eq. (13), in the phase space $\left(x_{n}^{l}, x_{n+1}^{l}\right)$, the sequence $\left(x_{0}^{l}, x_{1}^{l}, x_{2}^{l}, \ldots x_{n}^{l}, x_{n+1}^{l}, \ldots\right)$ generated by the $l$-th component $x^{l}$, is sampled chaotically, selecting $x_{n}^{l}$ every time the value $x_{n}^{m}$ of the $m$-th component $x^{m}$, is strictly greater than a threshold $\mathrm{T}$ belonging to the interval $[-1,1]$ of the real line.

The pseudo-code, for computing such chaotically sub-sampled iterates is: 


$$
\begin{aligned}
& X_{0}=\left(x_{0}^{1}, x_{0}^{2}, \ldots x_{0}^{p-1}, x_{0}^{p}\right)=\text { seed } \\
& n=0 ; q=0 ;
\end{aligned}
$$

do \{ while $n<N$

$$
\begin{aligned}
& \text { do }\left\{\text { while } x_{n}^{m}<T \text { compute }\left(x_{n}^{1}, x_{n}^{2}, \ldots x_{n}^{p-1}, x_{n}^{p}\right) ; n++\right\} \\
& \left.\left.\operatorname{compute}\left(x_{n}^{1}, x_{n}^{2}, \ldots x_{n}^{p-1}, x_{n}^{p}\right) ; \text { then } n(q)=n ; \bar{x}_{q}=x_{n(q)}^{1} ; n++; q++\right)\right\}
\end{aligned}
$$

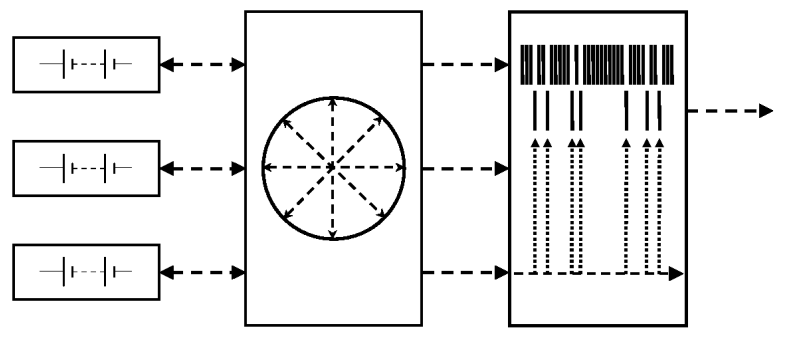

Fig. 7. Circuit of enhanced Chaotic Pseudo Random Number Generator (CPRNG) based on chaotic under-sampling.

This chaotic under-sampling is possible due to the independence of each component of the iterated points $X_{n}$ vs. the others [8]. We introduce the symbol on the right hand side of Fig. 7. in order to give a schematic representation of this chaotic under-sampling process.

\subsection{Mixer}

A second mechanism can improve the unpredictability of the pseudo-random sequence generated as above, using synergistically all the components of the vector $X_{n}$ instead of two.

Given $p-1$ thresholds $0<T_{1}<T_{2}<\cdots T_{p-1}<1$ forming a partition $J_{1}, J_{2}, \ldots, J_{p-1}$ of the interval $[-1,1]$, the pseudo-code, for computing such chaotically sub-sampled iterates is:

$$
\begin{aligned}
& X_{0}=\left(x_{0}^{1}, x_{0}^{2}, \ldots x_{0}^{p-1}, x_{0}^{p}\right)=\text { seed } \\
& n=0 ; q=0 ;
\end{aligned}
$$

do $\{$ while $n<N$

$$
\begin{aligned}
& \text { do }\left\{\text { while } x_{n}^{m} \in J_{0} \text { compute }\left(x_{n}^{1}, x_{n}^{2}, \ldots x_{n}^{p-1}, x_{n}^{p}\right) ; n++\right\} \\
& \text { compute }\left(x_{n}^{1}, x_{n}^{2}, \ldots x_{n}^{p-1}, x_{n}^{p}\right) ; \\
& \text { let } \left.\left.k \text { be such that } x_{n}^{p} \in J_{k} ; \text { then } n(q)=n ; \quad \bar{x}_{q}=x_{n(q)}^{k} ; n++; q++\right)\right\}
\end{aligned}
$$


We draw the symbol on the right hand side of Fig. 8. in order to give a schematic representation of the chaotic mixing process. For sake of simplicity we have only displayed a circuit with three 1-dimensional generators. However the mixing process runs better when more generators are coupled.

We can say that the design of mathematical circuit including couplers, samplers or mixers allows the emergence of complexity in chaotic systems which leads to randomness [10].

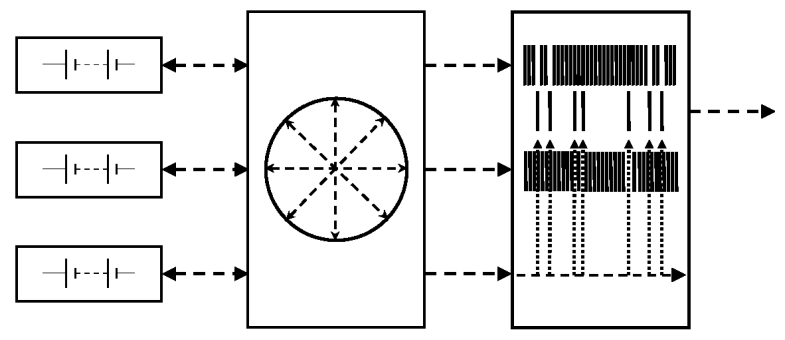

Fig. 8. Circuit of enhanced Chaotic Pseudo Random Number Generator (CPRNG) based

\subsection{Reducer}

We introduce now, another process which can directly provides random number without sampling or mixing, although it is possible to combine these processes with it. The idea underlying this process is to confine on $[-1,1]^{n}$ considered as a torus, a ring of $p$-coupled symmetric tent map (or logistic map) [3].

Consider the equation

$$
\left\{\begin{array}{c}
x_{n+1}^{1}=1-2\left|x_{n}^{1}\right|+k_{1} x_{n}^{2} \\
\vdots \\
x_{n+1}^{m}=1-2\left|x_{n}^{m}\right|+k_{m} x_{n}^{m+1} \\
\vdots \\
x_{n+1}^{p-1}=1-2\left|x_{n}^{p-1}\right|+k_{p-1} x_{n}^{p} \\
x_{n+1}^{p}=1-2\left|x_{n}^{p}\right|+k_{p} x_{n}^{l}
\end{array}\right.
$$

Where the parameters $k_{i}=\mp 1$. In order to confine the variables $x_{n+1}^{i}$ on this torus, we do, for every iteration, the transform 


$$
\left\{\begin{array}{l}
\text { if }\left(x_{n+1}^{j}<-1\right) \quad \text { add } \quad 2 \\
\text { if }\left(x_{n+1}^{j}>1\right) \quad \text { substract } 2
\end{array}\right.
$$

We design a new symbol: the reducer, on the right hand side of Fig. 9, in order to give a schematic representation of the projection of the variable on the torus. For sake of simplicity we have only displayed a circuit with three 1-dimensional generators. However this new pseudo-random number generator works better when more generators are coupled.

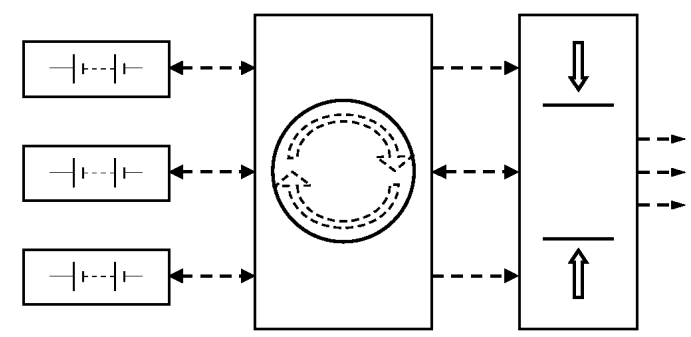

Fig. 9. Reducer for the circuit of Eq. (15) and the transform of Eq. (16).

\section{Design of mathematical chaotic circuits}

In the limited extend of this paper; it is difficult give examples of fully developed mathematical circuits. We first give the circuit of Cms-PRNG and then we use it for the design of new transmitter and receiver of cryptographic based chaos circuit.

\subsection{Chaotic multistream pseudorandom number generators (Cms- PRNG)}

It is possible to combine several equations in order to design chaotic multistream pseudo random number generators (Cms-PRNG) and processes in order to generate uncorrelated sequences of pseudo-random numbers, possessing a large number of keys for a cryptographic use. 


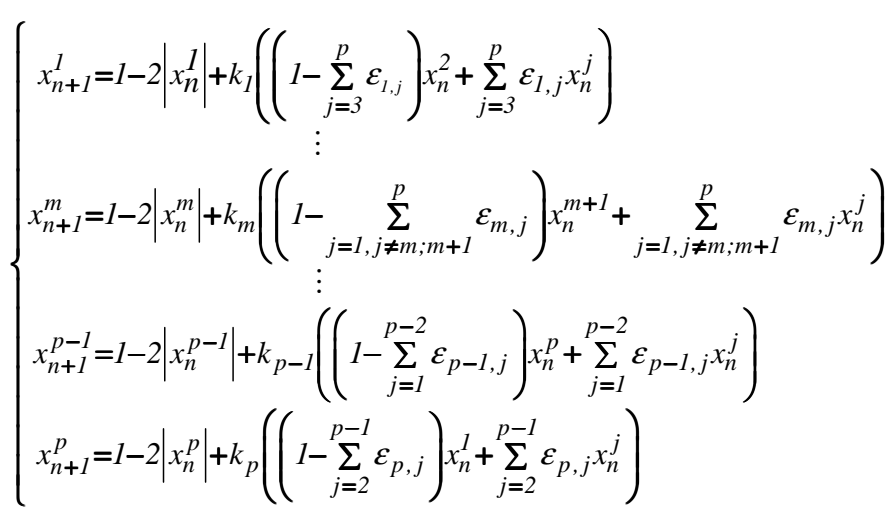

This is simply obtained by adding a full coupler as a keyer as shown in the circuit of Fig. 10, corresponding to Eq. (17) (with the reduction process of Eq.(16)).

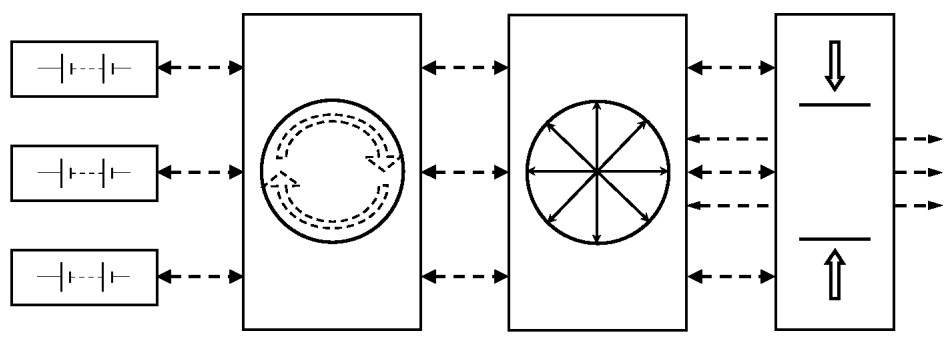

Fig. 10. Circuit of Cms-PRNG with only 3 streams.

\subsection{Noise-resisting cryptographic transmitter and receiver circuits}

The Cms-PRNG have been used for a novel ciphering method recently introduced in order to resist to noise which is always present during the transmission of the signal in any channel [1]. The main idea is to establish, between the transmitter and the receiver, a correspondence between the alphabet constituting the plain text and some intervals defining a partition of $[-1,1]$. Some realistic assumption about the noise boundedness allows restricting the bounds of the aforementioned intervals in order to precisely resist to the effects of the noise. An extra scrambling resorting to a co-generated chaotic sequence enhances the ciphering process. Then a new chaotic substitution method is developed: considering a chaotic carrier, belonging to the set of cogenerated and coupled pseudo-random chaotic sequences, the idea is to randomly/chaotically (in fact, this is determined by a second pseudorandom chaotic sequence) replace some elements of the carrier by a ciphered element (a letter here) of the message. At the receiver end, a copy of the Cms-PRNG, with the same parameters (hence we deal with a symmetrical ciphering method) 
allows to generate the necessary chaotic sequences and therefore to retrieve the initial message.

This process can be summarized in both circuits of Figs. 11 and 12. Due again to the limited extend of this paper, we cannot expand these figures in order to show the constituting symbols in each oval shaped region of the circuit. The originality of the method lies in the use of a chaotic pseudo-random number generator: several co-generated sequences can be used at different steps of the ciphering process, since they present the strong property of being uncorrelated. Each letter of the initial alphabet of the plain text is encoded as a subinterval of $[-1,1]$.

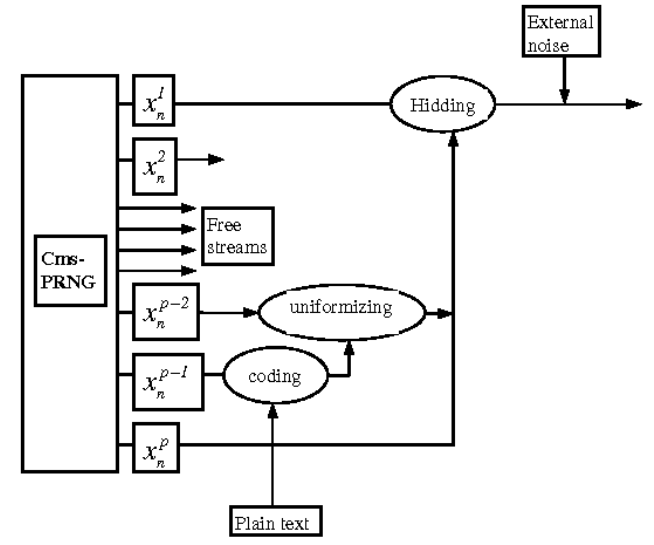

Fig. 11. Transmitter based on circuit of Fig. 10.

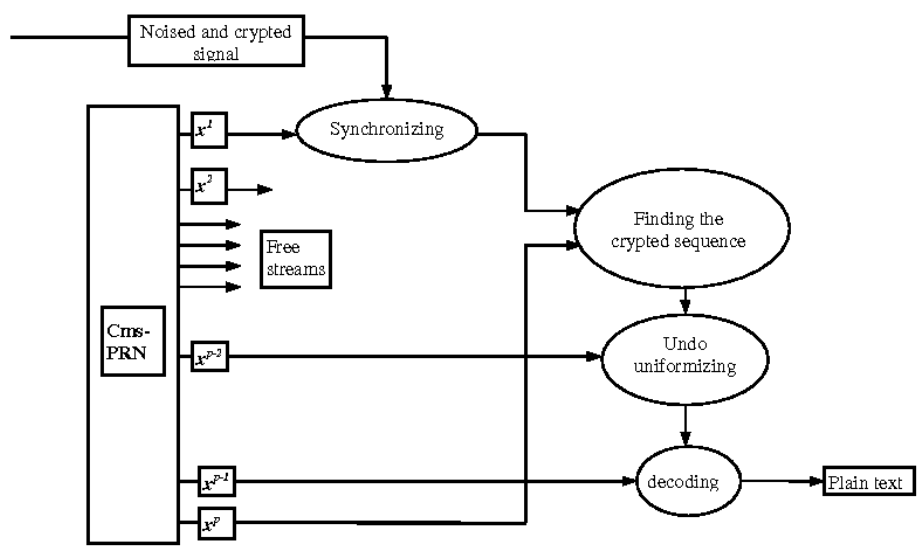

Fig. 12. Receiver of the coded signal.

The bounds of each interval are defined in function of the known bound of the additive noise. A pseudo-random sequence is used to enhance the complexity of the ciphering. The transmission consists of a substitution technique inside a chaotic carrier, depending on another cogenerated sequence. The efficiency of the pro- 
posed scheme is illustrated on some numerical simulations. As further work, some studies should be performed of several sets of unknown parameters, since with the considered Cms-PRNG with 10 states, the number of possible parameters amounts to 90 (the $\varepsilon_{i, j}$ and the $k_{i}$ ).

\section{Conclusion}

Following the worldwide tradition of use of Chua's circuits for various purposes, we have introduced the paradigm of chaotic mathematical circuitry which shows some similarity to the paradigm of electronic circuitry -the design of electronic circuits. This new paradigm allows, as an example, the building of new chaotic and random number generators.

Alongside to electronic circuits, the new theory of mathematical circuits allows many new applications in chaotic cryptography, genetic algorithms in optimization and in control [11], ... Due to the versatility of the new components we introduce, the combined operation of these chaotic mathematical circuits remains largely unexplored.

\section{References}

[1] Cherrier, E. \& Lozi, R., (2011), "Noise-resisting ciphering based on a chaotic multi-stream pseudo-random number generator", Proc. IEEE Conference Internet Technology and Secured Transactions (ICITST), 11-14 Dec. Abu Dhabi, 91-96.

[2] Chua, L. O., Kumoro, M. \& Matsumoto, T., (1984), “The Double Scroll Family”, IEEE Trans. Circuit and Systems, 32 (11), 1055-1058.

[3] Espinel, A., Taralova, I. \& Lozi, R. (2011), "Dynamical and Statistical Analysis of a New Lozi Function for Random Numbers Generation", in Proceeding of Physcon 2011, León, Spain, 5-8 September, IPACS open Access Electronic Library and Applications.

[4] Hénon, M., (1976), “A Two-dimensional mapping with a strange attractor”, Commun. Math. Phys., 50, 69-77.

[5] Kapitaniak, T., Chua, L. O. \& Zhong, G.-H., (1994), "Experimental hyperchaos in coupled Chua's circuits", IEEE Trans. Circuit and Systems, 41 (7), 499-503.

[6] Lozi, R. \& Chua, L.O., (1993), "Secure communications via chaotic synchronization II: noise reduction by cascading two identical receivers", Int. J. Bifurcation \& Chaos, 3 (5), 13191325.

[7] Lozi, R., (2006), "Giga-periodic orbits for weakly coupled tent and logistic discretized maps", In Modern Mathematical Models, Methods and Algorithms for Real World Systems, eds. A. H. Siddiqi, I. S. Duff \& O. Christensen, (Anamaya Publishers, New Delhi, India), 80124.

[8] Lozi, R., (2008), "New Enhanced Chaotic Number Generators", Indian Journal of Industrial and Applied Mathematics, Vol.1, no.1, 1-23.

[9] Lozi, R., (2011), "Complexity leads to randomness in chaotic systems", Mathematics in Science and Technology: Mathematical Methods, Models and Algorithms in Science and Technology. Proceedings of the Satellite Conference of ICM, 15-17 August 2010 Delhi, India 
(A.H. Siddiqi, R.C. Singh, P. Manchanda Eds.), World Scientific Publisher, Singapore, 93125 .

[10] Lozi, R., (2012), "Emergence of randomness from chaos", Int. J. Bifurcation \& Chaos, 22 (2), 1250021 (15 pages).

[11] Pluhacek, M., Senkerik, R., Davendra, D., Zelinka, I., (2013), "Designing PID controller for DC motor system by means of enhanced PSO algorithm with discrete chaotic Lozi map", Soft Computing Models in Industrial and Environmental Applications Advances in Intelligent Systems and Computing, 188, 475-483. 